\title{
Distributed channel assignment based on congestion information in wireless mesh network
}

\begin{abstract}
IEEE 802.11a protocol provides 12 non-overlapping channels. If nearby nodes operate on the same frequency channel, they can interfere with each other and produce congestion in the logical links. The use of Multi-Radio, Multi-Channel (MR-MC) can provide more coverage area due to multi-hop forwarding and offer more capacity by simultaneously operating on multiple radios. In this paper, we propose a dynamic, distributed channel assignment scheme for WMN, which is based on node queue length information. The proposed method assigns frequency channels based on queue threshold level which indicates the congestion status of the link. The algorithm does not allow the node to switch to the channel in which nearby nodes are operating. It also keeps record of previously congested channel to avoid assigning the same channel again. Simulation based experiment evaluated the performance in term of Round-Trip time (RTT).
\end{abstract}

Keyword: Channel assignment; Congestion; Interference; Multi-channel; Multi-radio; Wireless mesh network 